\title{
Microbial Ecotoxicology As An Emerging Research Subject
}

\author{
Ji-Dong Gu \\ Laboratory of Environmental Microbiology and Toxicology, School of Biological Sciences, The University of Hong Kong, Pokfulam Road, \\ Hong Kong SAR, P.R. China
}

\begin{abstract}
Microorganisms play an important role in cycling of elements of ecosystems, including a wide range of chemical pollutants from anthropogenic origin. These pollutants in ecosystems, particularly aquatic, and sediment and soils, are in different physical and chemical forms in association with the inorganic and organic constituents of the sediment and soils, resulting in variable availability of them to microorganisms for assimilation and transformation. A thorough and comprehensive knowledge of the physical and chemical states of them in the environments requires detailed information of both the bioavailable pollutant concentration and also the metabolic capability of the microorganisms to assess the ecological and environmental toxicity of these pollutants meaningfully. Apart from the primary role as decomposers, microorganisms are qualified to be sensitive indicators for environmental pollution, and ecological health and ecotoxicity of pollutants because of their very short generation time and quickly response to chemical pollutants than higher and large organisms. When used for testing with the same strain, different laboratories can generate high reproducible results to allow comparison of the data feasible, not mention the reduction in cost. Based on the current advances made on genomics analysis and bioinformatics, microbial genomes are easily assembled with the technologies available to providing useful transcriptomic and metabolic annotations, expression and prediction to allow advance toxicological to another level.
\end{abstract}

Keywords: Recalcitrant chemicals; biodegradation; mineralization; diminish of return; bioavailability; chemotaxis; metabolism; sequestration; microbial community; microbial toxicology; microbial ecotoxicology

*Correspondence to: Ji-Dong Gu, Laboratory of Environmental Microbiology and Toxicology, School of Biological Sciences, The University of Hong Kong, Pokfulam Road, Hong Kong SAR, P.R. China; E-mail: jdgu@hku.hk

Received: February 10, 2019; Accepted: February 22, 2019; Published Online: February 26, 2018

Citation: Ji-Dong Gu, 2019. Microbial Ecotoxicology As An Emerging Research Subject. http://doi.org/10.26789/AEB.2019.01.001.

Copyright: Microbial Ecotoxicology As An Emerging Research Subject.@ 2019 Ji-Dong Gu. This is an Open Access article distributed under the terms of the Creative Commons Attribution-Noncommercial 4.0 International License, permitting all non-commercial use, distribution, and reproduction in any medium, provided the original work is properly cited and acknowledged.

\section{Introduction}

Microbiota is the largest biomass widely occurring in different ecosystems including extreme environments with an array of metabolic activity contributing to the cycling of elements and supporting the function of ecosystems (Whitman et al., 1998). Though the active pool of organic matter is generally very small, the high turnover rate of their biochemical reactions makes them the active engine for the cycling of not only $\mathrm{C}$ but also N, P, and S. Microorganisms are ubiquitous and dynamics with high population. They also transform toxic chemicals through biochemical processes of these cycles (Alexander, 1999) and their endurance to pollutants allows them to be qualified as ecological indicators for environmental changes taking place and ecotoxicology.

Pollutants, both inorganic and organic in nature, are mainly a result of human activity and industrial production. Metals and metalloids in rock, minerals, and clay minerals are released at a very slow rate comparing to the anthropogenic driven acid mine drainage where $\mathrm{pH}$ value is extremely low and the concentration of various toxic metals and metalloids are unbelievably high, resulting in selection of microorganisms with capability to survive under such extreme conditions.
Most of the pollutants to human tend to be less or not watersoluble. Industrial chemicals of halogenated, polyaromatics, and aliphatics all have high molecular weights and very low water-solubility. Because of this, their concentration in water phase of the water/sediment is very low and, sometimes, has rarely detected in the aqueous phase in situ (Schwarzenbach et al., 2006). When considering the lithosphere, partition of organic pollutants between water and sediment is clearly different from those between octanol/water. In addition, humic substances in the ecosystem also have a significant role in changing the partition phenomenon and, more importantly, the toxicity of the pollutants. This situation is a fundamental question in the basic concept and understanding of ecotoxicology.

\section{Physical and Chemical States of Pollutants}

Assays based on pure chemicals in laboratory differ from the same amount of chemicals occurring in association with clay minerals because the latter affect the chemical bioavailability in a non-soluble condition. It is not difficult to understand that the toxic effects of the same concentration of a selec- 
tive pollutant differ between a solution in pure water and another water suspension containing clay minerals or organic matter (Dixon and Weed, 1977). Even though the total concentration of the chemical in both of them is identical by chemical analysis, the toxic effects to organisms is reduced significantly in the latter than the former. When a different clay mineral is used, montmorillonite to replace kaolinite, the observed toxicity is further lowered. Similarly, the presence of humic substances also changes the toxicity of pollutants in reconstituted system or natural ecosystems (Stotzky, 1986). Under field conditions of natural or polluted, similar phenomenon was reported in that lower toxicity is detected based on biological assessment in contrast to the chemical analysis for agrichemicals in agricultural soils (Alexander, 1999). Toxicity of pollutants also decreases over time of ageing in ecosystems. Such information is fundamental and also very important to ecotoxicological evaluation of environmental pollutants because apparent chemical concentrations by analytical chemistry are current used and reported for toxicological evaluation without any consideration of the ageing or the association with physical environmental constituents (Gu, 2018).

\section{Microorganisms in Natural Ecosystems}

Microorganisms are smaller in sizes, but they contribute to the nutrient cycling in the ecosystem enabling specific biochemical function of the different forms of life in a community (Whitman et al., 1998). Under natural condition, microorganisms form complex metabolic network and each individual member contributes to one specific small step in the overall biochemical transformation to achieve energy conservation for each member to growth (Amann et al., 1995). Among microorganisms, they sustain their own life cycle in the ecosystem independently for the specific step and also, in doing so, support the community by association with others through a number of relationships, including symbiotic, syntrophy, commensualism, antagonism, and mutualism etc. All these relationships show that life can be independent from each other, but more often dependent upon each others based on the relationship of metabolic network they contribute to, niche they share, and the specific environmental conditions to select specific members to be the dominant ones. Pollutants are one such selecting factor or pressure. This offers a wide variety of relationship and allows a further understanding of the in situ complex relationship among them. To understand microorganisms better in different ecosystems and contaminated sites, new research techniques and innovative thinking are indispensable to advance our knowledge and delineate the specific function of the microbial members in the ecosystem for a better protection of the environment.

\section{Ecotoxicity of Pollutants}

Toxicity of toxic chemicals and pollutants is assessed under laboratory conditions in an aqueous medium or application directly onto or into the target or indicator organisms, but such results can only be used as a reference for understanding of their toxicity to organisms through direct contact, not for in situ conditions of any natural ecosystems or contaminated sites. Because of this, concentration assayed by simply chemical extraction of selective solvent from an environmental matrice cannot be used reliably for projecting ecotoxicity evaluation of the target organisms living in the specific niche or ecosystems because of the significant deviation on meaning of concentrations between the chemical analysis and the effective to biological exposure (discussed above). A new approach should be proposed and used to obtain the chemical concentration that has an intrinsic meaning to the target organisms in the environment so that further advance of our understanding on the ecotoxicity of a chemical in the ecosystem can be made meaningfully with a higher level of confidence (Gu, 2014, 2016; Gu and Wang, 2013, 2014). One of the key information is to obtain the effective concentration, not the apparent analytical concentration by the current chemical analysis, in the environments and, as a result, the environmental or ecological toxicity of the chemical can be made on a more sound term and basis.

Ecological risk analysis, using data based on concentrations of environmental chemicals obtained analytically and also the toxicological data of chemicals based on selective model animals in laboratory system, makes toxicity prediction of the long-term exposure and in ecosystems (Yu and Gu, 2006; Yu and Gu, 2007a, 2007b). Such approach using extrapolation and various mathematical models has a major drawback in that the effective concentration or activity of the chemical in laboratory exposed to target organisms is not a meaningful one to organisms under conditions of ecosystem or complex system, e.g., sediment and soils. The concentration of a pollutant in a flask containing pure water and the pollutant will decrease after the addition of a clay mineral or sediment materials, such a decline can further progress to the stabilization of such change till an equilibrium state. This phenomenon shows clearly that interactions between the physical environment and the pollutants occur instantly and advance over time (Stumm and Morgan, 1996; Gu, 2016), so the bioavailability of pollutants is a changing value from the time of a spill and then afterward. This concept should be better recognized by ecotoxicologists and environmental toxicologists with training in chemistry or biology so that analytical concentrations of pollutants in the physical environment can be carefully obtained before a meaningful interpretation is made. To obtain the concentration by the current analytical procedures does not involve any consideration of the physical matrices to which the chemicals interact, so more effort shall be made on the effective concentration of the chemicals available to the organisms in situ than ex situ for ecotoxicological assessment. Because of this, a better understanding of 
the physical environment together with interactions between physical/chemical and biological components is required for a comprehensive understanding of environmental pollutants in the environment. Such knowledge will improve the prediction on ecotoxicology and toxic effects because more realistic data about toxic chemical concentration is obtained.

\section{Microorganisms for Ecotoxicology}

Microorganisms are small and have short generation time to allow fast growth to a high population density for research easily performed as standard testing organisms for a wide distribution globally. The unique characteristics of microorganisms in contrast to large organisms also allow the latter to be accepted as the organisms of choice to be used widely to lay a common foundation for calibration and meaningful comparison of data for further development and management policy (Cairns, 1983; Bitton and Dutka, 1986). Commercial success on product development using microorganisms can be made to provide reliable instrumentation on toxicity and carcinogenicity testing of chemicals. Microorganisms also have huge advantage to be indicators for ecological toxicity assays because they are small, short generation time, and at the low trophic level, responding to chemicals much rapidly (Mayfield, 1993). Bacteria can be shipped conveniently with less special care after lyophilized and sealed in ampules. On this direction, relatively far too less research has been conducted to advance and promote the utilization and acceptance of microorganisms as indicators for ecotoxicity and environmental quality in standard methods and protocols for international adoption (Gu and Wang, 2013, 2014).

Microorganisms have been successfully used in monitoring toxicity in several examples. The Ames test allows carcinogenicity of chemicals screened using the Salmonella spp. while Microtox uses luminescence of the bacterium Allivibrio fischeri (formerly Vibrio fishceri) to monitoring the growth and multiplication of an active population upon exposure to any chemicals for testing. It involves the use of a bioluminescence of bacterium emitting light through coupling luciferase with $\mathrm{NADH}$, and the intensity of the light emission is a direct function of the activity and viability of the cells, which are affected negatively by the exposure to chemical pollutants. By exposure of the target chemical at a gradient of concentrations, the results can be used to calculate $\mathrm{LC}_{50}$ or $\mathrm{LD}_{50}$ for toxicology evaluation use. The results of Microtox have high correlation with those obtained from other organisms including animals, making this test method widely accepted for environmental monitoring and regulation purposes (Bitton and Dutka, 1986; Cairns, 1983; Dutka and Bitton, 1986; Mayfield, 1993). These available tests, as examples, offer convenient procedures to assess toxicity in laboratory conditions and the results can be elaborated to environmental condition or ecosystem. These examples illustrate the advantages of utilizing microorganisms to generate comparable data globally to establish a solid basis for toxicological evaluation based on microorganisms. They are clear advantages over other testing organisms, including the barnacle amphitrite larvae and blue mussels for the more quick responses, easy handling and low cost. Obviously, microorganisms as testing organisms can be handled and shipped conveniently without difficulties and they can be preserved easily for a long period of time when not use. Large number of stock can be prepared for reference materials.

Microbial metabolism can be an important physiological indicator of the organism health and an essential indicator of the environmental conditions, especially exposure of toxicants. Phenotypic expression of Bacillus magneterium was reported with a decrease in the intensity of colorant synthesis and production in the culture medium when the concentrations of $\mathrm{Cr}^{6+}$ increase in the culture medium (Cheung and $\mathrm{Gu}, 2007$ ). Respiration of microorganisms can also be monitored sensitively and quantitatively through measuring the $\mathrm{CO}_{2}$ produced during the growth of microorganisms under specific conditions including presence of a pollutant. Commercial instrument is available for such measurement to allow higher reproducibility of the results and replicates. Microcalorimetry has been used to fulfill this goal in environmental monitoring and assessment of the ecological quality. In a similarly way, selective enzymes can be candidate for this kind of applications to indicate the environmental quality through a relationship between the enzyme activity and exposure of specific pollutant and different concentrations to establish a basis for this utilization.

Microbial ecological analysis of different ecosystems can also reveal important information on the microbial ecotoxicological information of specific impact from environmental change, pollution or alteration of ecological quality from human activity. Anaerobic ammonium oxidizing (anammox) bacteria can be a good indicator for human-related pollution, especially wastewater discharge monitoring and source tracing. In this regards, the different genera of anammox bacteria show clearly a niche specificity to pristine or polluted environments and this information can be used to deduce the environmental quality of a sample by analysis of the community composition of anammox bacteria (Han and $\mathrm{Gu}, 2015$ ). In addition, the same species of Scalindua zhenghei I, S. zhenghei II, and S. zhenghei III show more specific association with different part of the ocean environment from open ocean (pristine), shallow one, and costal wetland (wastewater contaminated) over a gradient of anthropogenic influence (Han and $\mathrm{Gu}, 2015$ ). This genuine approach on environmental quality assessment offers additional innovation to derived forensic information that is not possible from direct chemical analysis because the current pollution can be low due to dredging of contaminated sediment. Microbial analysis based on DNA and RNA extracted from sediment and water samples require only no more than 10 cells in a sample to trace the history because the ubiquity and persistence of microorganisms in the 
environment. This advantage of profiling microorganisms can also be extended to other functional group, such as ammonia-oxidizing archaea as another example (Cao et al., 2013).

\section{Conclusions}

Ecotoxicology and environmental toxicology need innovation and advance the knowledge for better understanding of the available concentrations of pollutants in situ to any target organisms for a meaningful assessment. In addition to the concentration, the indicator organisms shall allow reproducible testing results from different laboratories and easy distribution globally. Microorganisms have many advantages to offer for a range of tests to broaden the scope of the ecotoxicology information.

\section{Conflict of Interest}

Author declares that there is no conflict of interest in this research.

\section{Ethical approval}

This article does not contain any studies with human participants or animals performed by the author involved.

\section{References}

Alexander, M., 1999. Biodegradation and Bioremediation $\left(2^{\text {nd }}\right.$ ed.). Academic Press, San Diego, California.

Amann, R. I., Ludwig, W. and Schleifer, K.H., 1995. Phylogenetic identification and in situ detection of individual microbial cells without cultivation. Microbiol Rev, 59(1), 143-169. https://doi.org/ 10.1016/S0882-4010(95)90076-4

Bitton, G. and Dutka B.J., 1986. Toxicity testing using microorganisms. Vol. I. CRC Press, Boca Raton, Florida.

Cairns, J., 1983. Are single species toxicity tests alone adequate for estimating environmental hazard? Hydrobiology 100: 47-57. https://doi.org/ 10.1007/BF00394145

Cao, H., Auguet, J.C., and Gu, J.-D., 2013. Global ecological pattern of ammonia-oxidizing archaea. PLoS ONE, 8. https://doi.org/ 10.1371/journal.pone.0052853

Cheung, K.H. and Gu, J.-D., 2007. Mechanisms of hexavalent chromium detoxification by bacteria and bioremediation applications. International Biodeterioration \& Biodegradation 59: 8-15. https://doi.org/ 10.1016/j.ibiod.2006.05.002

Dixon, J.B. and Weed, S.B., 1977. Minerals in soil environments. Soil Science Society of America, Madison, WI. 948 pp

Dutka, B.J. and Bitton, G., 1986. Toxicity testing using microorganisms. Vol. II. CRC Press, Boca Raton, Florida.
Gu, J.-D., 2014. Assessment of ecosystem health and ecotoxicology through chemical analysis and modeling. Ecotoxicology 23 (4): 475-479. https://doi.org/ 10.1007/s10646-014-1206-x

Gu, J.-D., 2016. Biodegradation testing: so many tests but very little new innovation. Applied Environmental Biotechnology, 1(1): 92-95. https://doi.org/ 10.18063/AEB.2016.01.007

$\mathrm{Gu}$, J.-D., 2018. Bioremediation of toxic metals and metalloids for cleaning up from soils and sediments. Applied Environmental Biotechnology, 3(2): 48-51. https://doi.org/ 10.26789/AEB.2018.01.003

Gu, J.-D., and Wang, Y., 2013. A new era for geomicrobial ecotoxicology in environmental science research. International Biodeterioration \& Biodegradation 85: 345-346. https://doi.org/ 10.1016/j.ibiod.2012.06.024

Gu, J.-D., and Wang, Y., 2014. Geomicrobial ecotoxicology as a new subject in environmental sciences is proposed. Ecotoxicology 23 (10): 1823-1825. https://doi.org/ 10.1007/s10646-014-1359-7

Han, P., and Gu, J.-D., 2015. Further analysis of anammox bacterial community structures along an anthropogenic nitrogeninput gradient from the riparian sediments of the Pearl River Delta to the deep-ocean sediments of the South China Sea. Geomicrobiology Journal 32 (9): 789-798. https://doi.org/ 10.1080/01490451.2014.1001502

Mayfield, C.I. 1993. Microbial systems. pp. 9-27. In: P. Calow (ed.), Handbook of Ecotoxicology, Vol. I, Blackwell, London

Schwarzenbach, R.P., B.I. Escher, K. Fenner, T.B. Hofstetter, C.A. Johnson, U. von Gunten and B. Wehrli, 2006. The challenge of micropollutants in aquatic systems. Science 313: 10721077. https://doi.org/ 10.1126/science.1127291

Stotzky, G., 1986. Influence of soil mineral colloids on metabolic processes, growth, adhesion, and ecology of microbes and viruses. Pp. 305-428. In P.M. Huang and M. Schnitzer, eds. Interactions of Soil Minerals with natural Organics and Microbes. SSSA Special Publication No. 17. Soil Science Society of America, Inc., Madison, WI

Stumm, W. and Morgan J.J., 1996. Aquatic chemistry: chemical equilibria and rates in natural waters. ( $3^{r d}$ ed.), Wiley, New York. pp. 1022.

Whitman, W.B., Coleman, D.C. and Wiebe W.J.,1998. Prokaryotes: The unseen majority. Proc. Natl. Acad. Sci. USA 95: 6578-6583. https://doi.org/ 10.2307/44981

Yu X.Z. and Gu J.-D., 2006. Uptake, metabolism and toxicity of methyl tert-butyl ether (MTBE) in weeping willows. J Hazard Mater 137:1417-1423 https://doi.org/ 10.1016/j.jhazmat.2006.04.024

Yu X.Z. and Gu J.-D., 2007a. Accumulation and distribution of trivalent chromium and effects on hybrid willow (Salix matsudana Koidz $\times$ alba L.) metabolism. Arch Environ Contam Toxicol 52:503-511 https://doi.org/ 10.1007/s00244-006-0155-7

Yu X.Z. and Gu J.-D., 2007b. Metabolic responses of weeping willows to selenate and selenite. Env Sci Pollut Res 14:510517 https://doi.org/ 10.1065/espr2007.04.407 